\title{
Some Target Coverage Issues of Wireless Sensor Network
}

\author{
Raghvendra Kumar ${ }^{1}$, Prasant Kumar Pattnaik ${ }^{2}$, Priyanka Pandey ${ }^{3}$ \\ ${ }^{1}$ Department of Computer Science and Engineering, Lakshmi Narain College of Technology, Jabalpur, M.P., India \\ ${ }^{2}$ School of Computer Engineering, Kalinga Institute of Industrial Technology University, Bhubaneswar, Odisha, India \\ ${ }^{3}$ Department of Computer Science and Engineering, Takshshila Institute of Engineering and Technology, Jabalpur, M.P., India
}

\section{Email address:}

Raghvendraagrawal7@gmail.com (R. Kumar), patnaikprasantfcs@kiit.ac.in (P. K. Pattnaik), pandeypriyanka906@gmail.com (P.Pandey)

\section{To cite this article:}

Raghvendra Kumar, Prasant Kumar Pattnaik, Priyanka Pandey. Some Target Coverage Issues of Wireless Sensor Network. International Journal on Data Science and Technology. Vol. 2, No. 2, 2016, pp. 21-25. doi: 10.11648/j.ijdst.20160202.11

\begin{abstract}
Recent improvements in technologies have a considerable impaction advancing the state of wireless sensor networks, which constitute the platform of broad range of applications related to national Security, Habitat Monitoring, Environment observation and forecasting, Health Applications, Home and Office Applications. An important problem receiving increased consideration recently is the target cover age problem, which is concerned with random deployment of sensor nodes for monitoring the specific targets for maximum duration. These small sized sensor nodes have limited resources in terms of energy, memory, computational speed and bandwidth; and can't withstand extreme environmental conditions. Therefore, more number of sensor nodes is deployed than the specific requirements in order to improve fault to clearance of the sensor network. However, this high redundancy of sensor nodes can lead to excessive energy wastage. Since the nodes have limited energy resources and each sensor node requires a specific amount of energy to monitor a target, judicious energy management becomes an important concern of the Target Coverage Problem. Most existing works concentrate on scheduling sensors between sleep and active modes to maximize network life time while maintaining target coverage and consuming minimum energy. In this work, we try to develop an Energy Aware Target Coverage Protocol in which we have considered that if a target is within the receiving/ transmitting range of two or more sensors nodes, then only one of the sensor node may be initiated to cover the specific target while the other sensor nodes of that receiving/ transmitting range may not require to participate, which help to minimize participation of sensor nodes to cover the specific targets, there by consuming minimum energy. Our result analysis brings a considerable amount of energy saving without affecting the coverage problem.
\end{abstract}

Keywords: Wireless Sensor Network, Target Coverage, Energy Aware

\section{Introduction}

Wireless Sensor Networks have seen tremendous advancement in design and applications in the recent years. Wireless Sensor Networks (WSNs) involve deployment of huge number of wireless sensor nodes essentially for monitoring specific region of interest and collecting data. These collected data are then sent to the base station which acts like a control room and there further processing is done as per requirements. The rapid advancement of digital electronics and wireless communications has resulted in more rapid development of WSN technology. This rapid growth has resulted in focus being given into solving the challenges that this field has to face. One such challenge is to maximize the network life time of the network while the target nodes remain monitored constantly. This problem of maximizing the network lifetime while satisfying the coverage and also energy constraints (sensors are equipped with battery as the only power source and hence the energy constraint) is known as the Target Coverage Problem in Wireless Sensor Networks. Most of the works in target coverage deal with the problem of dividing the sensors into cover sets and scheduling these sets consecutively such as only sensors belonging in one set are active at any time, while the rest are inactive. Assuming a random sensor deployment and the fact that each sensor consumes the same amount of energy in each cover set the coverage problem is transformed to a problem of finding the optimal number of cover sets.

The emerging field of wireless sensor networks combines sensing, computation, and communication into a single tiny device. Through advanced mesh networking protocols, these devices form a sea of connectivity that extends the reach of cyberspace out into the physical world. As water flows to fill every room of a sub merged ship, the mesh networking connectivity will seek out and exploit any possible 
communication path by hopping data from node to node in search of its destination. While the capabilities of any single device are minimal, the composition of hundreds of devices of fers radical new technological possibilities. The power of wireless sensor networks lies in the ability to deploy large numbers of tiny nodes that as assemble and configure themselves. Usage scenarios for these devices range from real time tracking, to monitoring of environmental conditions, to ubiquitous computing environments, to monitoring of the health of structures or equipment. While often referred to as wireless sensor networks, they can also control actuators that extend control from cyber space into the physical world. The most straight forward application of wireless sensor network technology is to monitor remote environments for low frequency data trends. For example, a chemical plant could be easily monitored for leaks by hundreds of sensors that automatically form a wireless interconnection network and immediately report the detection of any chemical leaks. Unlike traditional wired systems, deployment costs would be minimal. Instead of having to deploy thousands of feet of wire routed through protective conduit, installers simply have to place quarter-sized device, at each sensing point. The network could be incrementally extended by simply adding more devices network or complex configuration. With the devices presented in this paper, the system would be capable of monitoring for anomalies for sever layers on a single to batteries. In addition to drastically reducing the installation costs, wireless sensor networks have the ability to dynamically adapt to changing environments. A adaptation mechanisms can respond to changes in network topologies or can cause the network to shift between drastically different modes of operation. For example, the same embedded network performing leak monitoring in a chemical factory might be reconfigured into a network designed to localize the source of a leak and track the diffusion of poisonous gases. The network could then direct workers to the safest path for emergency evacuation. Current wireless system only scratch the surface of possibilities emerging from the integration flow-power communication, sensing, energy storage, and computation. Generally, when people consider wireless devices they think of items such as cell phones, personal digital assistants, or laptops with 802.11. These items costs hundreds of dollars, target specialized applications and rely on the pre-deployment of extensive infrastructure support. In contrast, wireless sensor networks use small, low-cost embedded devices for a wide range of applications And do not rely on any pre-existing infrastructure. The vision is that

These devise will cost less that $\$ 1$ by 2005 . Unlike traditional wireless devices, wireless sensor nodes do not need to communicate directly with the nearest high-power control tower or base station, but only with their local peers. Instead, of relying on a pre-deployed infrastructure, each individual sensor or becomes part of the overall infrastructure. Peer-to-peer networking protocols provide a mesh-like interconnect to shuttle data between the thousands of tiny embedded devices in a multi-hop fashion. The flexible mesh architectures envisioned

Dynamically adapt to support introduction of new nodes or expand to cover a larger geographic region. Additionally, the system can automatically adapt to compensate for node failures.

The vision of mesh networking is based on strength in numbers. Unlike cell phone systems that deny service when too many phones are active in a small area, the interconnection of a wireless sensor network only grows stronger as nodes are added. As long as there is sufficient density, a single network of nodes can grow to cover limit less area. With each node having a communication range of 50 meters and costing less that $\$ 1$ a sensor network that encircled the equator of the earth will cost less than $\$ 1 \mathrm{M}$. It depicts a precision agriculture deployment an active area of application research. Hundreds of nodes scattered through out a field assemble together, establish a routing topology, and transmit data back to a collection point. The application demands for robust, scalable, low-cost and easy to deploy networks are perfectly met by a wireless sensor network. If one of the nodes should fail, a new topology would be selected and the overall network would continue to deliver data. If more nodes are placed in the field, they only create more potential routing opportunities.

\subsection{Wireless Sensor Networks}

The concept of wireless sensor

Networks are based on a simple equation: Sensing+ CPU+ Radio $=$ Thousands of potential applications As soon as people understand the capabilities of a wireless sensor network, hundreds of applications spring to mind. It seems like a straight forward combination of modern technology. However, actually combining sensors, radios, and CPU's in to an effective wireless sensor network requires a detailed understanding of the both capabilities and limitations of each of the underlying hardware components, as well as a detailed understanding of modern networking technologies and distributed systems theory. Each individual node must be designed to provide the set of primitives necessary to synthesize the interconnected web that will emerge as they are deployed, while meeting strict requirements of size, cost and power consumption. A core challenge is to map the overall system requirements down to individual device capabilities, requirements and actions. To make the wireless sensor network vision a reality, architecture must be developed that synthesizes the envisioned applications out of the underlying hardware capabilities. To develop this system architecture we work from the high level application requirements down through the low-level hardware requirements. In this process we first attempt to understand these to target applications. To limit the number of applications that we must consider, we focus on as to application classes that we believe are representative of a large fraction of the potential usage scenarios. We use this set of application classes to explore the system-level requirements that are placed on the overall architecture. From these system-level requirements we can then drill down into 
the individual node-level requirements. Additionally, we must provide a detailed background into the capabilities of modern hardware. After we present the raw hardware capabilities, we present a basic wireless sensor node.

\subsection{Sensor Network Application Classes}

The three application classes we have selected are: environmental data collection, security monitoring, and sensor node tracking. We believe that the majority of wireless sensor network deployments will fall into one of these class templates.

\subsection{Environmental Data Collection}

A canonical environmental data collection application is one where are search scientist wants to collect several sensor readings from as set of points in an environment over a period of time in order to detect trends and interdependencies. This scientist would want to collect data from hundreds of points spread throughout the area and then analyze the data offline. The scientist would be interested in collecting data over several months or years in order to look for long-term and seasonal trends. For the data to be meaningful it would have to be collected at regular intervals and then odes would remain at known locations. At the network level, the environmental data collection application is characterized by having a large number of nodes continually sensing and transmitting data back to a set of base stations that store the data using traditional methods. These networks generally require very low data rates and extremely long lifetimes. In typical usage scenario, the nodes will be evenly distributed over an outdoor environment. This distance between adjacent nodes will be minimal yet the distance across the entire network will be significant. After deployment, the nodes must first discover the topology of the network and estimate optimal routing strategies. The routing strategy can then be used to route data to a central collection points. In environmental monitoring applications, it is not essential that the nodes develop the optimal routing strategies on their own. Instead, it may be possible to calculate the optimal routing topology outside of the network and then communicate the necessary information to the nodes as required. This is possible because the physical topology of the network is relatively constant. While the time variant nature of RF communication may cause connectivity between two nodes to be intermittent, the overall topology of the network will be relatively stable. Environmental data collection applications typically use tree-based routing topologies where each routing tree is rooted at highcapability nodes that's ink data. Data is periodically transmitted from child node to parent node up the treestructure until it reaches the sink. With tree-based data collection each node is responsible for forwarding the data of all its descendants. Nodes with a large number of descendants transmit significantly more data than leaf nodes. These nodes can quickly become energy bottlenecks. Once the network is configured, each node periodically samples its sensors and transmits its data up the routing tree and back to the base station. For many scenarios, the interval between these transmissions can be on the order of minutes. Typical reporting periods are expected to be between 1 and 15 minutes; while it is possible for networks to have significantly higher reporting rates. The typical environment parameters being monitored, such as temperature, light intensity, and humidity, does not change quickly enough to require higher reporting rates. In addition to large sample intervals, environmental monitoring applications do not have strict latency requirements. Data samples can be delayed inside the network for mode rate periods of time without significantly affecting application performance. In general the data is collected for future analysis, not for real-time operation. In order to meet life time requirements, each communication event must be precisely scheduled. The senor nodes will remain majority of the time; they will only wake to transmit or receive data. If the precise schedule is not met, the communication events will fail. As the network ages, it is expected that nodes will failover time. Periodically the network will have to recon figure to handle node or link failure or to redistribute net workload. Additionally, as the researchers learn more about the environment they study, they may want to go in and insert additional sensing points. In both cases, the reconfigurations are relatively in frequent and will not represent a significant amount of the overall system energy usage. The most important character is tics of the environmental monitoring requirements are long life time, precise synchronization, low data rates and relatively static topologies. Additionally it is not essential that the data be transmitted in real-time back to the central collection point. The data transmissions can be delayed inside the network as necessary in order to improve network efficiency.

\section{Target Coverage Problem of WSN}

Target Coverage Problem is one of the challenges of Wireless Sensor Network. It is concerned with the maximization of sensor network life time while performing the task of monitor ring the specific targets by a set of randomly dispersed sensor nodes (shown in Figure1). Considering a number of targets (say $\mathrm{r}_{1}, \mathrm{r}_{2}, \mathrm{r}_{3} \ldots . . \mathrm{r}_{\mathrm{m}}$ ) whose location are already pre-determined, a set of sensor nodes (say $\mathrm{s}_{1}, \mathrm{~s}_{2} \ldots \ldots \ldots \mathrm{s}_{\mathrm{n}}$ ) are redundantly deployed with the help of a aircraft to keep track of these specific targets, assuming that a sensor node covers the target if the Euclidean distance between the sensor node and the target is smaller or equal to the sensing range of the node. Each of these nodes requires energy to monitor the targets. Since the nodes have limited battery life, efficient utilization of the available energy resources becomes important for performing the coverage task for a maximum period of time. There for energy conservation becomes a critical issue while tracking the targets.

A number of targets with known locations are considered that need to be continuously observed (covered) and a large number of sensors are randomly deployed closed to the 
targets. A central data collect or node is also considered which is called as the base station (BS).This BS can be the cluster head into a more general, cluster-based frame work. Sensed data might be processed locally by the sensors or at the $\mathrm{BS}$, from where it is aggregate forwarded to the user. It is assumed the sensors have location determination capabilities (e.g. GPS). As the number of sensors deployed in the field is assumed to be greater than the optimum need to perform the required task, an important energy-efficient method consists in scheduling the sensor nodes activity to alternate between active state and sleep state. It is considered that a sensor node can go to the sleep mode when the node is not scheduled to perform the sensing task.

\section{Challenges}

Wireless Sensor Nodes while covering the specific targets may suffer from some challenges such as:

- Sensor nodes are prone to failure due to extreme environmental conditions.

- The topology of a sensor network changes very frequently.

- Sensor nodes are limited in power, computational capacities, and memory.

- Wireless Sensor Networks processing sensitive data are facing the risks of data manipulation, data fraud and sensor destruction or replacement.

- WSN deals with real world problems where the sensed data must be delivered within fixed time limits. However most protocols fail to meet the deadlines.

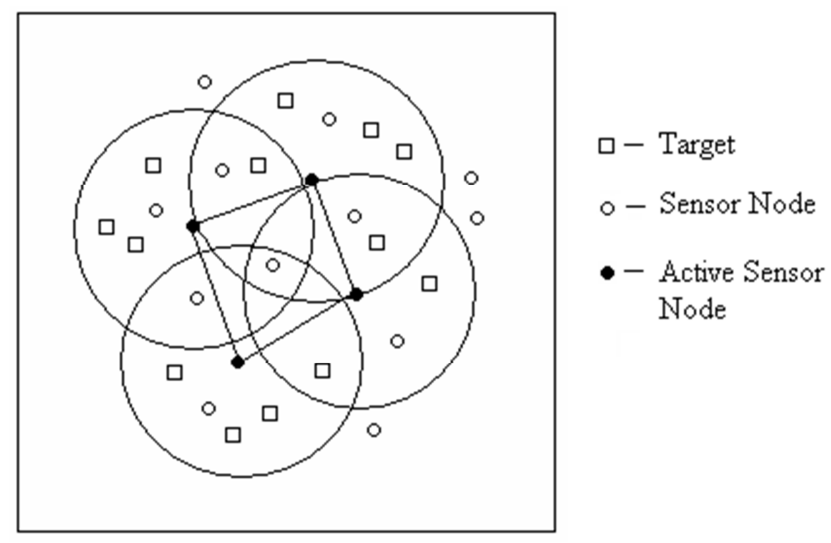

Figure 1. A Scenario of Target Coverage Problem.

There fore more number of sensors than the specific requirements is deployed to improve the fault to clearance. However, this high redundancy of sensor nodes can lead to excessive energy vast agenda also the maintenance of batteries of these nodes is not a simple task. So while covering the targets, minimum consumption of energy must be take to consider a achieve coverage for a maximum duration. An energy saving technique is to alter these nodes between high energy consumption active mode and low energy consumptions sleeping mode. The nodes that are actually performing the coverage task are inactive mode that consumes a considerable amount of energy while the rest nodes may enter into sleep mode which consumes negligible amount of energy. The latter nodes can be reentered into active mode when needed and the former may go into sleep mode. This scheduling of nodes may resulting efficient energy conservation.

In the Figure1, four sensor nodes represented by small filled circles are in active mode covering the targets represented by small squares. Rest sensor nodes represented by small circle share in sleep mode.

\section{Some Target Coverage Strategies}

Some of the Strategies to cover a specific set of target share mentioned below:

\subsection{Activating all the Sensors at a Time}

In order to cover all the specific targets, the simplest method is to activate all the sensor nodes deployed for coverage at a time. All the activated sensor nodes co-operatively keep track of the specific number of targets with known location. However, this simultaneous activation of sensor nodes to cover the required targets may make the nodes exhaust of their energy at a time. So, only for a limited amount of time they would be able to perform the coverage task.

\subsection{Formation of Disjoint Set Covers of Sensor Nodes}

An energy efficient method to cover all the targets is to make the sensor nodes alter between active and sleep modes. During active mode, a sensor node is capable of keeping track of the target sand their important information. If a sensor node is having no task, it may enter in to sleep mode which consumes a negligible amount of energy. So, some disjoint set covers of active sensor nodes may be made in such a manner that each set cover is covering all the targets. These set covers are activate done after another till the sensor nodes are out of their energy. So, rather than making all the sensor nodes active at a time, they can be partitioned into a number of disjoint set covers with each set cover capable of monitoring the targets.

Cardei and Du address the target coverage problem where disjoint sensors model disjoint set covers, such that every cover completely monitors all the target points. These set share then activated successively, and while the current sensor set is active, all other nodes are in the sleep mode. The goal of this approach is to determine a maximum number of disjoint sets, as this has a direct impact on conserving sensor energy resources as well as on prolonging the network life time.

\subsection{Formation of Non-disjoint Set Covers of Sensor Nodes}

Another energy efficient method for target cover age is to make the sensor nodes be part of more than one set cover. With the sensor nodes altering between the active and sleep modes. Several non disjoint set cover so active sensor nodes are made to activate successively where each set cover is capable of keeping track of all the specific targets until the energy exhaustion of sensor nodes. This procedure is much 
more energy efficient as compared to disjoint set cover method of target coverage, thus maximizing the network life time to some more extent

Cardei, Thai, Liand $\mathrm{Wu}$ proposed an efficient method to extend the sensor network life time by organizing the sensors into a maximal number of set covers that are activated successively. Only the sensors from the current active set are responsible for monitoring all targets and for transmitting the collected data, while all other nodes are in a low-energy sleep mode. By allowing sensors to participate in multiple sets, the problem formulation increases the network life time compared with related work.

\subsection{Partial Target Coverage}

Till now we have mentioned full coverage of targets which ensures that all their targets are to be covered where the sensor nodes are divided into cover sets, with the sensors in each set are capable of monitoring all targets in the field. By activating one cover set at a time, the sensor network life time can be extended. Information collected by a subset of targets may also be beneficial. A number of set cover of sensor nodes may be activated for partial coverage of the targets, not necessarily covering all the targets. The gathered information about a subset of targets by the sensor nodes may prove to be very important where the main objective is to maximize the life time of the network.

Zorbas, D., Glynos, D.\& Douligeris analyzed the problem of connected partial target coverage where cover sets are allowed to monitor a subset of the targets at any point in time, taking into account several characteristics of the problem, such as the specific topology parameters, the coverage status of the sensors as well as their remaining battery life.

\subsection{K-Coverage}

Ink coverage $(\mathrm{k} \geq 1)$ approach, aminimal subset of the sensor nodes is determined that can keep track of the required targets, with each target being covered by at least $\mathrm{k}$ sensor nodes. The activated nodes collectively gather information about the specific targets and send it to the base station.

Zong heng Zhou, Samir Das, Himanshu Gupta analyzed the problem of k-coverage where a set of Sensors nodes M are deployed such that each target in the sensor network is covered by at least $\mathrm{K}$ different sensors in set $\mathrm{M}$.

\section{Conclusion}

Target Coverage Problem is concerned with the maximization of the network lifetime while the target nodes remain monitored constantly. Wireless sensor nodes keeping track of targets are battery powered, there for the network lifetime through a power aware node organization is highly desirable. An efficient method for energy saving is to schedule the sensor node activity such that every sensor alternates between sleep and active state. One solution is to organize the sensor nodes in set covers, such that every cover completely monitors all the targets. These covers are activated in turn, such that at a specific time only one sensors responsible for sensing the targets, while all other sensors are in the sleep state. In certain cases information collected by a subset of sensor nodes can also be beneficial. In this paper we have discussed all these cover the specified targets with known locations by a set of randomly dispersed sensor nodes.

\section{References}

[1] Sanjaya Kumar Padhiand Prasant Kumar Pattnaik, "A Novel Distributed Protocol for Randomly Deployed Clustered Based Wireless Sensor Network", Journal of Theoretical and Applied Information Technology, Vol 15. No. 1, 2010.

[2] X. - Y. Li, P. - J. Wan, and O. Frieder, "Coverage in Wireless Adhoc Sensor Networks", IEEE Transactions on Computers, Vol 52 (2002), pp 753-763.

[3] V. Raghunathan, C. Schurgers, S. Park, and M. B. Srivastava, "Energy-Aware Wireless Micro sensor Networks", IEEE Signal Processing Magazine, 19 (2002), pp 40-50.

[4] Zorbas, D., Glynos, D. \& Douligeris, C, "Connected partial target coverage and network life time in wireless sensor networks", Wireless Days (WD), $20092^{\text {nd }}$ IFIP, pp. 1-5.

[5] D. Tianand N. D. Georganas, "A Coverage-Preserving Node Scheduling Scheme for Large Wireless Sensor Networks," Proc. First ACM Int'l Workshop Wireless Sensor Networks and Applications, pp. 32-41, 2002.

[6] S. Meguerdichian, F. Koushanfar, M. Potkonjak, and M. Srivastava, "Coverage Problems in Wireless Adhoc Sensor Networks", IEEE Info com (2001), pp 1380-1387.

[7] X. Wang, G. Xing, Y. Zhang, C. Lu, R. Pless and C. D. Gill, "Integrated Coverage and Connectivity Configuration in Wireless Sensor Networks," Proceedings of the $1^{\text {st }}$ International Conference on Embedded Networked Sensor Systems, Los Angeles, 2003, pp. 28-39.

[8] M. Cardei and D. - Z. Du, "Improving Wireless Sensor Network Life time through Power Aware Organization", ACM Wireless Networks, Vol. 11, No. 3, pp. 333-340, May 2005.

[9] K. Kar and S. Banerjee, "Node Placement for Connected Coverage in Sensor Networks", Proc. Of WiOpt 2003: Modeling and Optimization in Mobile AdHoc and Wireless Networks (2003).

[10] Yu Gu, Jie Li, Baohua Zhao, and Yusheng Ji, "Target Coverage Problem in Wireless Sensor Networks: A Column Generation Based Approach," in Proceedings of $6^{\text {th }}$ IEEE International Conference on Mobile Ad-hoc and Sensor Systems (2009). 